\title{
Entre faits et fiction : l'instruction de la demande d'asile en Allemagne et en France
}

In between facts and fictions: Asylum claims' procedures in Germany and France

Johanna Probst

\section{(2) OpenEdition}

12 Journals

\section{Édition électronique}

URL : http://journals.openedition.org/conflits/18243

DOI : $10.4000 /$ conflits. 18243

ISSN : 1777-5345

Éditeur :

CCLS - Centre d'études sur les conflits lilberté et sécurité, L'Harmattan

Édition imprimée

Date de publication : 31 décembre 2011

Pagination : 63-80

ISBN : 978-2-296-96617-8

ISSN : 1157-996X

Référence électronique

Johanna Probst, «Entre faits et fiction : l'instruction de la demande d'asile en Allemagne et en

France », Cultures \& Conflits [En ligne], 84 | Hiver 2011, mis en ligne le 15 mars 2013, consulté le 30

mars 2021. URL : http://journals.openedition.org/conflits/18243 ; DOI : https://doi.org/10.4000/ conflits. 18243 


\section{Entre faits et fiction : I'instruction de la demande d'asile en Allemagne et en France}

\section{Johanna PROBST}

Johanna Probst est doctorante en sociologie. Elle prépare une thèse en cotutelle avec l'Université de Strasbourg (Laboratoire Cultures et Sociétés en Europe) et la Philipps-Universität Marburg (Institut für Soziologie / Zentrum für Konfliktforschung). Ses recherches portent sur la problématique des migrations forcées et de l'asile.

\section{Comprendre le processus décisionnel}

T a migration forcée est un phénomène dont la signification centrale pour le - monde d'aujourd'hui apparait quasi quotidiennement à différentes échelles géopolitiques. «Nous avons besoin d'une discussion sociologique qui pointe l'importance de la migration forcée dans la société contemporaine et dans les actuels processus de changement ${ }^{1}$ ", réclame le sociologue australien Stephan Castels. Selon cet auteur, la migration forcée structure les relations diplomatiques internationales et constitue un enjeu tant pour les pays d'origine que pour les pays de destination des demandeurs d'asile. Au fil du XXI siècle, nombreux pays du continent européen se sont mus de pays émetteurs en pays receveurs de réfugiés politiques ${ }^{2}$. À l'issue de la Seconde Guerre mondiale, la communauté internationale se dote d'un instrument juridique essentiel pour assurer la protection des personnes persécutées : la Convention de Genève de 1951. L'interprétation de plus en plus étroite de la définition du réfugié y figurant, ainsi que l'élaboration par beaucoup d'États signataires (notamment européens) de dispositions juridiques restrictives affaiblissent la portée de ce texte de loi ${ }^{3}$. Avec la création de l'espace Schengen, la nécessité

1. «We need a sociological argument that points to the significance of forced migration in contemporary society and in current process of change ». Castels S., "Towards a Sociology of Forced Migration and Social Transformation”, Sociology, vol.37, n¹, 2003, p. 16 (traduction par l'auteure).

2. Laurain, M., «Les réfugiés dans le monde. Que croire, que faire ? ", Études, vol.397, n ${ }^{\circ}$, 2002, pp. 31-42.

3. Voir par exemple : Decourcelle A., Julinet S., Que reste-t-il du droit d'asile ?, Paris, L'Esprit 
d'une coopération des pays européens en matière d'immigration et d'asile se fait sentir ${ }^{4}$. Depuis les années quatre-vingt-dix, de nombreux efforts visent l'établissement d'un dispositif d'asile harmonisé et commun à tous les États membres de l'Union européenne (UE). Au vu de l'évolution de la situation géopolitique internationale et de la nature des conflits mondiaux, les dispositions de la Convention de Genève sont jugées inadaptées et incomplètes par les États européens. À travers l'adoption récente de plusieurs directives, l'UE veut offrir aux demandeurs d'asile ayant réussi à gagner le territoire européen des conditions matérielles et juridiques similaires dans chaque État membre. Tout en précisant les qualités du « réfugié » conventionnel, la directive dite « qualification 5 » crée un statut de protection subsidiaire permettant de protéger des personnes dont le profil ne cadre pas avec la définition de ce premier. Parallèlement à ce nouvel élargissement ${ }^{6} \mathrm{du}$ champ de protection, un système de contrôle militarisé des frontières extérieures de l'UE visant à rendre le plus difficile possible l'accès au territoire européen - et donc aussi à la procédure d'asile - est mis en place ${ }^{7}$. Au-delà, une base de données biométriques dénommée Eurodac ${ }^{8}$, assurant le fichage de tous les migrants déposant une demande d'asile auprès d'un État membre est constituée, conformément au règlement Dublin II 9.

Malgré cette communautarisation des politiques d'asile, la procédure administrative de demande lors de laquelle sont censées être appliquées ces nouvelles normes juridiques est toujours prise en charge par les appareils bureaucratiques des divers États nationaux. De nombreuses enquêtes constatent de manière peu surprenante que d'importantes différences entre les dispositifs d'asile nationaux persistent après la transposition des textes européens

frappeur, 2000 ; Fassin D., Morice A., Quiminal C. (eds.), Les lois de l'inhospitalité. Les politiques de l'immigration à l'épreuve des sans-papiers, Paris, La Découverte, 1997.

4. Voir par exemple : Carlier J.Y., "Introduction : le développement d'une politique commune en matière d'asile ", in Dias Urbano de Sousa C., De Bruycker P. (eds.), L'émergence d'une politique européenne d'asile, Bruxelles, Bruylant/Editions de l'ULB, 2004, pp. 1-14 ; Legoux L., "Changements et permanences dans la protection des réfugiés », Revue européenne des migrations internationales, vol.20, $\mathrm{n}^{\circ} 2,2004$, pp. 9-22.

5. Directive du Conseil 2004/83/CE, 29 avril 2004 (Directive concernant les normes minimales relatives aux conditions que doivent remplir les ressortissants des pays tiers ou les apatrides pour pouvoir prétendre au statut de réfugié ou les personnes qui, pour d'autres raisons, ont besoin d'une protection internationale, et relatives au contenu de ces statuts).

6. La création de la protection subsidiaire n'aurait, selon des auteurs comme Thomas Spijekeboer, pas été nécessaire si l'instrument juridique de la Convention de Genève n'avait pas préalablement été démantelé : Spijkerboer T., « Full circle ? The personal scope of international protection in the Geneva Convention and the draft on qualification ", in Dias Urbano de Sousa C. \& De Bruycker P. (eds.), L'émergence d'une politique européenne d'asile, Bruxelles, Bruylant, 2004, pp. 167-181.

7. Voir par exemple : Le Pors A., Le Droit d'asile, Paris, PUF, 2005 ; Guiraudon V., « Les effets de l'européanisation des politiques d'immigration et d'asile ", Politique européenne, n ${ }^{\circ} 31$, 2010, pp. 7-32 ; Valluy J., «L'exportation de la xénophobie de gouvernement. De la politique européenne des frontières à la répression dans les pays limitrophes ", in Fassin D. (ed.), Les nowvelles frontières de la société française, Paris, La Découverte, 2010, pp. 175-196..

8. Règlement du Conseil CE n 2725/2000, 11 décembre 2000.

9. Règlement du Conseil CE n 343/2003, 18 février 2003. 
en loi nationale ${ }^{10}$. Dans ce contexte de redéfinition du droit d'asile au niveau européen, de tentatives d'harmonisation intérieure accompagnées d'un verrouillage des frontières extérieures, il semble intéressant de comparer la mise en ouvre concrète de la procédure de demande d'asile et du processus décisionnel qu'elle implique par les États membres. Le présent article se base sur une recherche empirique comparative réalisée au cours de l'année 2010 au sein du Bundesamt für Migration und Flüchtlinge (BAMF - «Office fédéral de la migration et des réfugiés ») et de l'Office français de protection des réfugiés et apatrides (OFPRA) ${ }^{11}$. Si les politiques d'asile nationales et européennes ont largement été analysées et commentées dans la littérature scientifique, le déroulement concret du traitement administratif des demandes d'asile a assez peu été étudié. Ceci semble entre autres lié aux traditionnelles réticences des institutions de l'asile à accueillir des chercheurs ou journalistes. C'est certainement grâce à une récente politique d'ouverture et de transparence 12 que ces institutions m'ont autorisée à mener une enquête sociologique au plus près des agents administratifs instruisant la demande d'asile.

Dans une perspective interactionniste et dans la lignée des travaux sociologiques sur "l'État au guichet » et la relation administrative 13, cette recherche a pour objectif de comprendre comment les agents parviennent à articuler la loi universelle et le cas individuel afin de produire une décision

10. Voir par exemple : Neumayer E., “Asylum recognition rates in Western Europe. Their determinants, variations, and lack of convergence”, Journal of Conflict Resolution, $\mathrm{n}^{\circ} 49,2005$, pp. 43-66 ; Hailbronner K., Normes minimales relatives aux conditions pour pouvoir prétendre au statut de réfugié ou à la protection internationale et contenu de ces statuts. Évaluation (résumé) de la transposition de la directive 2004 et proposition pour un Régime européen commun d'asile, 2008.

11. Suite à une activité de bénévole au sein d'une association de soutien aux demandeurs d'asile et à une enquête réalisée sur les réfugiés reconnus en France (2008/2009), mon intérêt s'est progressivement porté vers l'appareil administratif et juridique prenant en charge la procédure de demande d'asile en France et en Allemagne. La réalisation d'une recherche empirique au BAMF et à l'OFPRA a pu être négociée avec des responsables institutionnels. Respectivement 24 (BAMF) et 17 (OFPRA) entretiens semi-directifs ont été menés auprès d'agents instructeurs (j'appelle « instructeurs » les agents administratifs chargés de la réalisation de l'entretien avec le demandeur et de l'instruction de son dossier donnant lieu à une proposition de - décision) et respectivement 12 et 10 avec leurs supérieurs hiérarchiques et des agents de divers services institutionnels. Les entretiens ont globalement duré entre 60 et 90 minutes. Dans chaque institution ont été effectuées cinq observations directes d'entretiens entre demandeurs et agents. Le matériau empirique comprend également des observations d'audiences au sein des instances juridiques d'appel ainsi qu'un petit nombre d'entretiens avec des magistrats et des avocats intervenant dans le contentieux de l'asile en France et en Allemagne.

12. Ce constat correspond aux allégations des responsables des services de communication du BAMF et de l'OFPRA et se confirme par le peu de difficultés que nous avons rencontrées pour négocier l'accès au terrain.

13. Voir par exemple : Weller J. M., L'État an guichet. Sociologie cognitive du travail et modernisation administrative des services publics, Paris, Desclée de Brouwer, 1999 ; Fassin D., "Charité bien ordonnée. Principes de justice et pratiques de jugement dans l'attribution des aides d'urgence ", Revue française de sociologie, vol.42, n³, 2001, pp. 437-475; Warin P., Les Dépanneurs de justice. Les "petits fonctionnaires " entre qualité et équité, Paris, L.G.D.J., 2002 ; Dubois V., La vie au guichet. Relation administrative et traitement de la misère, Paris, Economica, 1999. 
administrative. Une interrogation sur la capacité décisionnelle des agents paraît particulièrement pertinente au regard des spécificités de la matière administrative de l'asile, à savoir la dureté des faits exposés par les requérants pour justifier de leur demande et l'enjeu important que celle-ci implique pour eux. Au-delà, la production de la décision administrative s'inscrit dans un contexte d'incertitude et d'ignorance, dû à l'absence quasi totale de preuves matérielles et au soupçon institutionnel généralisé à l'égard de la parole des demandeurs ${ }^{14}$. La question de la crédibilité des déclarations des demandeurs occupe une place centrale dans l'instruction des demandes d'asile. La hiérarchie institutionnelle incite les agents à vérifier pointilleusement la véracité des récits présentés. Dans les deux institutions, les taux d'accord ${ }^{15}$ ont, durant plusieurs années, oscillé entre $5 \%$ et $10 \%$. Les porte-parole du BAMF et de l'OFPRA (ainsi que de nombreux représentants gouvernementaux des deux pays) ont tendance à expliquer l'extrême faiblesse de ces taux par le caractère infondé et peu crédible d'une large majorité de demandes. Notons qu'il existe dans chacun des pays étudiés un contrôle juridique des décisions administratives : en France, les annulations prononcées par la Cour nationale du droit d'asile (CNDA) font quasiment doubler le taux d'accord ${ }^{16}$.

Malgré l'élévation du taux d'accord final grâce au contrôle juridictionnel, les pratiques décisionnelles (peu généreuses) mises en œuvre par les appareils bureaucratiques des États entrent en contradiction avec la grande valeur symbolique que les États français et allemand proclament attacher au droit d'asile. Ce décalage suscite de multiples questions chez les chercheurs et d'autres acteurs extra-institutionnels ${ }^{17}$. Au regard de cette situation, une observation directe du travail administratif et une interrogation sur les critères et mécanismes officieux d'admissibilité apparait essentielle pour saisir les tenants et aboutissants du processus décisionnel. Dans une perspective microsociologique, le taux d'accord doit être vu comme le résultat d'une longue série de décisions singulières dont chacune met en jeu plusieurs facteurs et acteurs. Cette enquête appréhende la procédure d'instruction du point de vue de ceux qui y détiennent le pouvoir de décision. L'attention se focalisera sur la situation d'entretien qui met en face à face l'agent et le demandeur, sur le compor-

14. Voir par exemple : Belorgey J., «Le droit d'asile en perdition ", Recueil Alexandries, 2007, mis en ligne mai 2007, URL : http://reseau-terra.eu/article598.html ; Welge I., Untersuchung zur Entscheidungspraxis des Bundesamtes für Migration und Flüchtlinge in Fällen eritreischer Asylantragsteller, 2006 ; Rousseau C., Foxen P., "Le mythe du réfugié menteur : un mensonge indispensable ?", L'Évolution psychiatrique, n`71, 2006, pp. 505-520 ; Jubany O., "Contructing truth in a culture of disbelief. Understanding asylum screening from within", International Sociology, vol.26, n¹, 2011, pp. 74-94.

15. Le taux d'accord représente la proportion de décisions positives parmi l'ensemble des décisions prises sur une année donnée.

16. En raison d'importantes différences dans le déroulement de la procédure de recours allemande, qui est prise en charge par les tribunaux administratifs locaux, aucun taux d'annulation global comparable au taux français ne peut être avancé ici.

17. Voir par exemple : Schuster L., "A Comparative Analysis of the Asylum Policy of Seven European Governments”, Journal of Refugee Studies, vol.13, n ${ }^{\circ}$, 2001, pp. 118-132 ; Valluy J., Rejet des exilés. Le grand retournement du droit d'asile, Paris, Éditions du Croquant, 2009. 
tement des demandeurs tel qu'il est observé et décrit par les instructeurs ainsi que sur la question cruciale de la crédibilité prédominant l'interaction. Il s'agit donc de proposer une analyse critique de la production du taux d'accord : estil avant tout conditionné par la « qualité de la demande d'asile », comme le suggèrent les acteurs institutionnels ? Est-il au contraire, et selon la thèse de Jérôme Valluy ${ }^{18}$, la conséquence d'une intention politique cherchant à limiter le nombre de personnes annuellement admises au titre de l'asile ? Quelle est la marge de manœuvre des agents instructeurs? Quel est leur « ethos professionnel » ? Et quel rôle joue la présentation de soi et la performance des demandeurs devant l'institution dans le processus décisionnel ? Voici les questions qui seront abordées au fil de cet article.

\section{Le profil socioprofessionnel contrasté des agents}

Comparant les institutions allemande et française de l'asile, le premier constat qui saute aux yeux du chercheur est le contraste entre le profil socioprofessionnel de leurs agents respectifs, en particulier de ceux en charge de l'instruction. Les données livrées par la suite se réfèrent à la population totale des instructeurs en poste en 2010 dans chacune des institutions, sachant que les échantillons de l'enquête sont à cet égard parfaitement représentatifs. Les agents instructeurs des deux institutions se distinguent nettement à propos de quatre variables : leur formation, leur âge, leur ancienneté dans l'institution et leur sexe. Les « officiers de protection instructeurs » de l'OFPRA sont majoritairement issus de filières universitaires juridiques ou (géo)politiques et souvent spécialisés en droit international ou humanitaire. On y trouve également des diplômés de disciplines littéraires, linguistiques ou bien de sciences sociales. La majorité d'entre eux a autour de trente ans et leur ancienneté moyenne dans l'institution s'élève à environ cinq ans ${ }^{19}$. Dans la population des instructeurs de l'OFPRA, les femmes sont largement surreprésentées (74\%) 20. Le profil des Entscheider ("décideurs », selon l'intitulé officiel) du BAMF apparaît comme tout à fait opposé. D’abord, ils ressortent exclusivement de formations juridiques ou administratives et n'ont initialement aucune spécialisation relative à la thématique de l'asile. Ils ont en moyenne 51 ans, et 18 ans d'ancienneté dans le métier. $65 \%$ des instructeurs du BAMF sont des hommes.

Interrogés sur les raisons de leur candidature à ce poste de travail, les instructeurs de l'OFPRA mettent souvent en avant un intérêt pour la matière administrative de l'asile, pour le contact avec des personnes issues d'autres cultures et mentionnent des motifs moraux renvoyant à une envie d'aider des

18. Ibid.

19. Malheureusement, nous ne disposons pas de chiffres précis pour le cas français. Il s'agit donc ici d'estimations basées sur nos observations de terrain.

20. Malgré cette surreprésentation des femmes, nous allons continuer à parler des « instructeurs français/de l'OFPRA » dans la forme masculine englobant donc bien les deux sexes. 
gens en détresse et d'honorer l'engagement humanitaire noble de la France. Leurs collègues allemands insistent surtout sur des motifs matériels : la proximité du lieu de travail par rapport au lieu de vie, la rémunération, mais aussi la grande autonomie et la liberté décisionnelle offerte par le poste de travail. Les différences que l'on constate concernant l'arrière-fond éducatif des instructeurs de l'OFPRA et du BAMF laissent supposer ce qui se confirme dans les entretiens : travailler dans l'administration de l'asile représente pour les premiers la prolongation d'un intérêt préalable pour des questions géopolitiques, interculturelles, humanitaires ou migratoires. Les seconds affirment quasi unanimement ne pas avoir eu d'intérêt particulier pour la problématique de l'asile ou des migrations avant d'entreprendre le métier d'instructeur. Celui-ci semble davantage être vu comme une activité administrative quelconque. Tandis que les instructeurs de l'OFPRA ont, dans leur vie antérieure, fréquemment travaillé bénévolement ou professionnellement dans le milieu associatif ou militant, leurs homologues du BAMF ont souvent une expérience professionnelle dans un autre domaine administratif comme les chemins de fer, l'agence du travail ou encore l'administration militaire.

Il faut mentionner ici que la quasi-totalité des instructeurs en poste au BAMF aujourd'hui ont été recrutés (y compris au sein d'autres administrations) au début des années quatre-vingt-dix - époque à laquelle le nombre de demandes d'asile déposés en Allemagne a atteint des records (au-delà des 400000 demandes annuelles) ${ }^{21}$. Puisque la demande a continuellement baissé depuis 1992 (pic de 438191 demandes), l'office allemand s'est rapidement retrouvé avec un sureffectif d'instructeurs. Les instructeurs du BAMF aujourd'hui proches de la retraite relèvent donc tous d'une même génération ayant connu « l'état d'exception » (selon leur propre description) des années quatre-vingt-dix et ont fait preuve d'une grande stabilité professionnelle. Inversement, un turn-over important assure le renouvellement constant des agents instructeurs de l'OFPRA, comme le reflète la faible ancienneté moyenne. Le départ «précoce » (aux yeux des dirigeants) de bon nombre d'entre eux semble en partie s'expliquer par de simples raisons de carrière et de projet professionnel, mais aussi par certaines frustrations liées aux conditions du travail d'officier de protection instructeur, notamment les exigences considérables de productivité. Bien que les instructeurs allemands expriment plus ouvertement un mécontentement à l'égard de certaines consignes, pressions et contraintes émanant de la hiérarchie institutionnelle, rares sont ceux qui effectuent ou envisagent un abandon du métier.

Dans la suite de l'article, nous allons nous intéresser à la manière dont les instructeurs remplissent leur tâche professionnelle. L'instruction des dossiers comprenant l'entretien avec les demandeurs d'asile constitue le cœur de celleci. L'analyse se concentrera sur les stratégies et procédés que les agents déve- 
loppent face à la difficulté de devoir prendre des décisions dans une situation caractérisée par la pénurie permanente d'informations, le doute et l'incertitude 22 .

\section{Les difficultés de l'entretien institutionnel}

Les instructeurs peuvent être considérés comme les «street-level bureaucrats » du dispositif d'asile (selon l'expression de Michael Lipsky 23). Ce sont eux qui sont, en première ligne, confrontés à la tâche épineuse de juger de la véracité des récits présentés et de qualifier juridiquement les faits qu'ils peuvent établir comme vraisemblables. Au cours de leur expérience professionnelle d'instructeur semble s'opérer une érosion progressive de la confiance fondamentale caractéristique des relations sociales quotidiennes. Des informations qui, d'ordinaire, font rarement l'objet de doutes, en viennent à être largement dénuées de leur évidence dans le cadre de l'entretien avec le demandeur d'asile : «Le problème est que vous ne savez même pas qui vous avez en face de vous. Ils arrivent ici avec une ribambelle d'enfants et vous ne savez même pas si ce sont leurs enfants! [...] Vous voyez à quel point c'est difficile quand les identités ne sont pas claires. Ils peuvent prétendre quelque chose, mais comment voulez-vous savoir si c'est vrai ? ", déplore un instructeur allemand, tandis qu'un autre confirme que «dans la procédure d'asile, les noms ne sont que bruit et fumée $24 »$. Cette attitude de doute radical semble s'amplifier au fil des années d'exercice du métier d'instructeur. Elle est ainsi bien plus prégnante et fréquente chez les agents allemands dont l'ancienneté moyenne dans le métier est environ quatre fois plus élevée que celle de leurs collègues français. Selon la description des agents, l'intensité du doute est progressive : ils décrivent une évolution d'une attitude quelque peu «naïve » vers une «méfiance raisonnable».

Chancelant sur le sol vacillant du droit d'asile, les instructeurs sont obligés de construire des repères stables et d'élaborer une méthode leur permettant de « rendre décidable l'indécidable 25 ». Selon Jérôme Valluy, les juges de la CNDA opèrent la «substitution d'une question traitable, celle de la conviction, à une question qui ne l'est pas en situation de double ignorance, celle de la vérité $26 »$. Ce constat ne semble que partiellement valable pour les agents de l'OFPRA et du BAMF, dans la mesure où ceux-ci cherchent à éviter des appréciations démesurément subjectives par intime conviction. Dans les pro-

22. Scheffer T., Asylgewährung. Eine ethnographische Verfabrensanalyse, Stuttgart, Lucius \& Lucius, 2001.

23. Lipsky M., Street-Level Bureancracy. Dilemmas of the Individual in Public Services, New York, Russell Sage Foundation, 2010.

24. Traduction littérale de l'expression allemande «Schall und Rauch » qui désigne une illusion, quelque chose dont la réalité ne peut être supposée.

25. Scheffer T., "Kritik der Urteilskraft - Wie die Asylprüfung Unentscheidbares in Entscheidbares überführt », in Oltmer J. (ed.), Migration Steuern und Verwalten, Göttingen, Vandenhoeck \& Rurpecht, 2003 (traduction par l'auteure).

26. Ibid., p. 118. 
cédures de sélection, les agents institutionnels poursuivent le but d'une objectivation maximale des décisions. Leurs efforts pour obtenir des preuves matérielles des faits de persécution ou des identités allégués par les demandeurs sont cependant fréquemment contrecarrés par les tentatives de ces derniers de se dérober à ces procédures de vérification. La période où les instructeurs attribuaient une grande importance aux documents probants (mandats d'arrêt, dépositions de plaintes, cartes de membre de parti, articles de journaux, certificats médicaux attestant de traces de torture) semble révolue depuis que l'authenticité des documents présentés apparaît comme de plus en plus douteuse. À défaut de pouvoir la vérifier, l'OFPRA a largement revu à la baisse la valeur de preuve des documents. Le BAMF cependant n'hésite pas à engager des moyens financiers importants pour faire établir des expertises par des spécialistes externes. En cas de doute sur la région d'origine d'un demandeur (et à condition que cette question soit cruciale pour la décision sur sa demande), les agents allemands peuvent par exemple commander une analyse linguistique : pour une rémunération d'environ $400 €$ un linguiste analyse un enregistrement de la voix du demandeur afin d'en déduire sa véritable région d'origine.

En l'absence de preuves matérielles, les instructeurs misent tout sur l'entretien pour discerner le bien-fondé d'une demande. Lors de celui-ci, ils testent la capacité du demandeur à présenter un récit paraissant à leurs yeux cohérent, logique et probable. Comme l'observe Scheffer ${ }^{27}$, ce test prend largement la forme d'un contrôle de connaissances orienté en fonction des faits biographiques que le demandeur invoque. Sa réalisation suppose que l'instructeur dispose lui-même d'un savoir détaillé concernant le contexte sur lequel il interroge le demandeur. Ce savoir s'érige parfois en certitude inébranlable et repère absolu pour l'établissement des faits. J'ai ainsi pu observer une instructrice affirmer devant un candidat hésitant sur ses réponses : "Je connais mieux votre pays d'origine que vous, Monsieur ». Malgré leurs connaissances précises sur les zones géographiques qu'ils traitent, les raisonnements des instructeurs sont souvent biaisés par leur propre expérience de vie dans un pays occidental qui leur sert de repère dans l'appréciation du caractère possible ou vraisemblable de certains faits relatés dans le récit. Ainsi, une instructrice allemande argumente : «Je ne pense pas que les services de sécurité des pays d'origine se focalisent sur des jeunes filles politiquement actives. Ce ne sont pas les personnes dangereuses! Ils n'ont pas suffisamment d'effectifs pour cela! Ils ne peuvent pas sonner cinq fois par semaine chez elles pour enquêter ». Dans une décision du BAMF, on trouve un raisonnement du même type : «Eu égard aux normes morales en Iran, il est inexplicable qu'une femme non mariée fréquente un homme marié, comme ce dernier le soutient. Ne serait-ce qu'en raison de ce qu'il explique concernant le contexte familial de son prétendu nouvel amour, il n'est pas compréhensible qu'une femme célibataire entreprenne effectivement une relation - même platonique - avec 
lui ». Cette réflexion qu'un instructeur français fait à un demandeur turc qui déclare avoir milité dans un parti communiste révolutionnaire et qui se présente à l'entretien institutionnel avec un blouson Adidas constitue un troisième exemple :

Question : « Porter un blouson de marque Adidas n'est pas un peu en contradiction avec la révolution et les idées de cette dernière ? " Réponse : «Je suis étudiant de l'université, je comprends votre point de vue mais je ne suis pas si borné que ça. Il faut une certaine présentabilité. Et il fallait que je prenne soin de ma tenue en venant ici; c'est pas parce que je suis révolutionnaire que je dois venir comme un clochard ici ».

Notons néanmoins que nombre d'instructeurs sont conscients de leurs présupposés personnels et occidentaux susceptibles de les induire en erreur. La méthode du test de connaissances présente pour les instructeurs une autre limite. Puisqu'ils ont pu être acquis lors d'un apprentissage par cœur et non lors d'une expérience authentique, les connaissances des demandeurs ne sont pas considérées à elles seules comme une garantie de véracité. Les instructeurs attendent donc une «personnalisation du récit » qui leur montrerait que le demandeur a lui-même vécu ce dont il témoigne. Une instructrice française me rapporte l'anecdote suivante :

«Et j’ai une jeune fille qui m’a dit: "quand mon papa est arrivé, il avait été détenu, qu'est-ce qu'il sentait mauvais !” C'est bête, elle rougissait en le disant... Mais ce sont les impressions sur les sens, qui peuvent faire penser qu'ils ont vécu ça. Parce qu'on ne pense pas à inventer une chose pareille. »

Il devient dès lors apparent que la préparation des demandeurs avant l'entretien contrecarre certaines techniques employées par les instructeurs pour parvenir à évaluer la crédibilité, dans la mesure où ils dénaturent leur récit et leur autoprésentation. Tandis que les agents institutionnels déplorent le manque de «fraîcheur » des demandeurs, ces derniers voient une préparation soigneuse, voire une performance quasi théâtrale devant l'instructeur, comme seule possibilité pour se donner toutes les chances de réussir l'épreuve institutionnelle.

\section{L'introuvable « vrai réfugié politique »}

Les réalités que les demandeurs d'asile présentent devant les guichets institutionnels échappent souvent à la rigidité des catégories juridico-administratives. La création de la protection subsidiaire reflète un processus d'adaptation des dispositions juridiques aux évolutions des conflictualités mondiales, dans la mesure où elle témoigne de la prise en compte de problématiques qui ne 
relèvent pas de la «traditionnelle » persécution politique : la menace par des acteurs non étatiques, la guerre civile, la problématique du genre, de l'homosexualité, etc. ${ }^{28}$. Les instructeurs ont, sur le principe, accueilli favorablement cette évolution. Ils s'accordent néanmoins pour dire que leur tâche professionnelle s'en est trouvée complexifiée. Les « anciens » (à savoir tous les instructeurs du BAMF et une minorité parmi ceux de l'OFPRA) sont les plus sceptiques quant à l'évolution actuelle, regrettant un éloignement de l'esprit initial du droit d'asile qui aurait selon eux été crée avant tout pour protéger des personnes poursuivies par des États totalitaires et dictatoriaux. Les problématiques inclues depuis dans le champ de protection appartiennent cependant plutôt au domaine des conflits claniques, ethniques, voire privés. Établir la crainte d'un mariage forcé est, selon les instructeurs, bien plus difficile que d'établir une arrestation pour activités oppositionnelles, dans la mesure où cette première problématique relève entièrement de la vie privée et familiale des personnes. Selon un agent d'encadrement, «c'est très déstabilisant pour les OP [officiers de protection] » qui « se posent beaucoup de questions pour savoir si c'est vrai que cette femme est victime de violences, si ce jeune homme a reçu des coups de feu parce qu'il aimait sa cousine... ». Les problématiques désormais prises en compte se marient mal avec la représentation du « vrai réfugié politique » que s'étaient forgée les instructeurs ayant connu l'époque précédant l'introduction de la protection subsidiaire. Une certaine nostalgie de cette figure incarnant des valeurs de courage et de force morale apparaît en filigrane de leurs propos.

L'évaluation des dossiers de demande semble toujours fonctionner à l'aide d'un modèle du «vrai réfugié » nécessitant et méritant protection, que chaque agent administratif se construit en croisant son expérience personnelle avec la norme juridique et jurisprudentielle, la doctrine institutionnelle et ses connaissances sur les pays de provenance. Les instructeurs moins anciens semblent en effet avoir intégré les critères juridiques pour l'attribution de la protection subsidiaire dans leur représentation de la personne nécessiteuse d'une protection qu'il s'agit de reconnaître. L'exclusion nette de tout ce qui ressemble à un motif migratoire économique constitue cependant toujours une constante traversant les diverses conceptions individuelles $\mathrm{du}$ " vrai réfugié ». Les rencontres exceptionnelles avec ces derniers provoquent chez les instructeurs une excitation joyeuse et leur « rappellent pourquoi ils font ce métier ». Ainsi, un instructeur allemand m'explique qu'il trouve « incroyablement satisfaisant d'avoir parfois un vrai persécuté. Un vrai indemnise pour cinquante menteurs. Il est très agréable et bienfaisant de faire un entretien avec un vrai ». Nonobstant la peur de " passer à côté » d'un tel dossier, beaucoup d'instructeurs sont assez convaincus de savoir le reconnaître : «...et quand je

28. Voir par exemple : Wihtol de Wenden C., « Migrations et droits de l'homme en Europe », Études internationales, vol.24, n¹, 1993, pp. 163-176 ; Decourcelle A., Julinet S., Que reste-til du droit d'asile ?, Paris, L'Esprit frappeur, 2000. 
tombe sur un vrai militant politique, un vrai, là je me dis : ah, ça se voit la différence ! C’est frappant ! 29 ». La représentation du «vrai réfugié » renvoie donc non seulement à ses qualités de victime d'un système oppressif auquel il a voulu s'opposer, mais aussi à l'impression de sincérité et d'honnêteté qu'il produit chez l'instructeur.

\section{Se présenter à l'institution}

Selon le soupçon nourri au sein de l'institution, le modèle du « vrai réfugié » inspire les histoires racontées et la présentation réalisée par la masse des "faux ». Au-delà des critères juridiques traçant les contours de la figure du réfugié, les qualités immanentes distinguant le premier des seconds sont sujettes aux définitions variables des agents. Ces critères apparaissent ainsi comme aussi incertains que le contenu précis des histoires susceptibles de mener à un accord et font perpétuellement l'objet de négociations intra-institutionnelles et de spéculations extra-institutionnelles. Par peur que les demandeurs calquent leurs récits sur la doctrine institutionnelle, celle-ci est gardée comme un secret d'État. Pour cette même raison, les décisions positives ne sont pas motivées. Les savoirs, conseils et recommandations qui circulent à l'extérieur de l'institution se fondent donc largement sur des hypothèses plus ou moins vagues concernant «ce qu'il faut faire ou dire » pour être reconnu. En effet, un vaste réseau d'assistance comprenant des avocats, des acteurs associatifs, des compatriotes déjà sur place ainsi que les passeurs accompagnant le voyage des réfugiés s'est constitué au fur et à mesure du durcissement des contrôles et des règlementations des États européens. Les divers conseils et informations que le demandeur reçoit durant le temps d'attente précédant la convocation l'incitent à réfléchir à la manière appropriée de se présenter à l'institution, à préparer ses formulations et à se munir des connaissances supposées nécessaires ${ }^{30}$. Il est alors peu étonnant que les déclarations des demandeurs au moment de l'entretien apparaissent à l'instructeur, quelle que soit leur teneur en vérité, comme dépourvues de spontanéité et d'authenticité, comme convenues et finalement comme peu crédibles. De nombreux instructeurs admettent d'ailleurs que l'institution finit certainement par rejeter injustement certains demandeurs dont le récit semblait artificiel malgré la réalité des craintes de la personne.

29. Données aimablement fournies par Karen Akoka.

30. Sur la préparation des récits avant l'entretien institutionnel, voir : Noura K., « Travail de construction de la crédibilité, processus d'évaluation et catégorisation des motifs de persécution : étude de cas, les femmes réfugiées provenant d'ex-URSS », Revue Asylon(s), vol.1, mis en ligne octobre 2006, http://reseau-terra.eu/article504.html ; D’Halluin E., «Passeurs d'histoire. L'inconfort des acteurs associatifs impliqués dans l'aide à la procédure d'asile ", in Fassin D. (ed.), Les nouvelles frontières de la société française, Paris, La Découverte, 2010, pp. 363-383; Kobelinsky C., L'accueil des demandeurs d'asile. Une ethnographie de l'attente, Paris, Éditions du Cygne, 2010. 
Le comportement des demandeurs devant l'institution doit ainsi être considéré, en amont de tout jugement moral, comme une adaptation aux attentes supposées de leurs évaluateurs, comme une mise en conformité avec l'image du réfugié que ceux-ci récompensent par l'attribution du statut, comme un effort pour réussir l'épreuve de l'entretien. Il s'agit d'un mécanisme ayant déjà été décrit par de nombreux sociologues. Michael Lipsky met en lumière le processus d'apprentissage et d'inculcation du rôle de «client » ou d'usager d'une institution qui dispense une prestation convoitée ${ }^{31}$. Ainsi, Vincent Dubois observe qu'un allocataire aura tendance à se présenter à la Caisse d'allocations familiales comme un «bon pauvre » nécessiteux et méritant ${ }^{32}$. La théorie goffmanienne ${ }^{33}$ semble utile à la description de ces comportements : selon un exemple cité par cet auteur, le pauvre cherche à exhiber les signes de son dénuement devant le travailleur social susceptible de lui accorder des aides. La façade personnelle est ainsi construite en fonction des attentes institutionnelles. Si des tiers ont assuré au demandeur que l'instructeur attend qu'un réfugié pleure quand il raconte la mort d'un proche, le demandeur s'efforcera de pleurer ; s'il faut soi-disant produire un mandat d'arrêt pour prouver être recherché, le demandeur se débrouillera pour présenter un tel document; si la crédibilité d'un récit de torture augmente quand le demandeur en porte des traces visibles, ce dernier peut être poussé à les inscrire lui-même sur son corps. Dans la recherche d'une conformité avec l'image du réfugié, l'automutilation présente la pratique la plus extrême et désespérée qui heureusement reste très rare. Elle exprime cependant la dérive d'un système qui pousse ses sujets à créer la réalité qui légitimera leur demande. Pour donner un exemple fictif et caricatural : en vendant leur maison, en s'endettant auprès de leur famille pour payer le passeur, en abandonnant entièrement une vie antérieure, les demandeurs ne peuvent effectivement plus rentrer chez eux sans risquer l'exclusion, la misère matérielle, le blâme familial. En détruisant leurs documents d'identité, ils rendent leur expulsion plus difficile. Certains parmi eux entreprennent dans leur pays d'accueil un engagement politique en faveur d'une organisation persécutée dans leur pays d'origine, cherchent à documenter et rendre visible leurs activités sur Internet et créent ainsi une situation qui justifie en effet des craintes en cas de retour. Or, de telles transformations de la réalité ne sont pas toujours à l'initiative des réfugiés. Parfois, des demandeurs ayant essayé durant plusieurs années de faire reconnaître leur cas comme digne de protection et ayant essuyé refus sur refus finissent par en devenir fous ou dépressifs - et obtenir un droit au séjour justement pour cette raison.

31. Lipsky M., op. cit., pp. 66-67.

32. Dubois V., op. cit.

33. Goffman E., La Mise en scène de la vie quotidienne. La présentation de soi, Paris, Les Éditions de Minuit, 1973. 
La remise en cause des récits présentés par les demandeurs en vue de l'obtention du statut de réfugié n'a pas été inventée dans les années quatre-vingt. Comme le montre Gérard Noiriel pour le cas français, un examen pointilleux des demandes (de droit au séjour de manière générale) et une méfiance envers les récits des demandeurs se pratiquaient déjà à une période antérieure à la Convention de Genève, durant les années vingt et trente ${ }^{34}$. Or, ces pratiques prennent un nouvel essor au cours des années quatre-vingt, et ce dans la majorité des pays européens ${ }^{35}$. Comme le montre Karen Akoka dans sa thèse en cours ${ }^{36}$, on cesse dès lors de parler de « réfugiés » pour adopter la désignation de «demandeur d'asile », glissement sémantique révélateur du doute à l'égard de la qualité de réfugié des migrants qui se présentent aux guichets de l'OFPRA. Corollaires l'un de l'autre, la (ré)apparition de ce doute semble tout aussi incontestable que l'utilisation de plus en plus fréquente de récits d'emprunt et l'occurrence d'autres comportements considérés comme frauduleux dans la procédure d'asile. À en croire les représentants institutionnels et gouvernementaux, c'est suite à la découverte de ces faits que l'administration commence à considérer les demandes d'un oil plus suspect. Cependant, la chronologie de cette relation causale pourrait tout aussi bien être inverse : peut-être que la présentation de récits (partiellement) inventés ou de documents contrefaits représente la réaction à un durcissement des pratiques administratives, à une élévation du niveau d'exigences en termes de crédibilité et à une attitude généralement moins favorable à la reconnaissance des réfugiés. Sans vouloir trancher sur les raisons de celle-ci, l'introduction du doute et de la méfiance radicale dans la procédure d'asile déclenche un processus de surenchère : à mesure que les institutions augmentent leurs exigences en termes de crédibilité et de gravité des faits allégués par les demandeurs, ces derniers adaptent leurs dossiers à ces exigences; à mesure que les demandeurs présentent des faits biographiques et des documents inauthentiques, les institutions instaurent des procédures de vérification et de contrôle sévères. "Nos décisions sont à l'image de la demande : stéréotypées et convenues », me disait un instructeur français. Or il est également possible que ce soit, au contraire, la demande qui soit à l'image des décisions stéréotypées et convenues de l'institution.

Il n'est pas besoin de savoir « qui a commencé » pour constater que le processus de surenchère $a$, de fait, mené à une situation où la demande d'asile se

34. Noiriel G., Réfugiés et sans-papiers. La République face au droit d'asile XIXe-XXe siècle, Paris, Hachette, 1999, p. 192 et suivantes.

35. Voir par exemple : Herbert U., Geschichte der Ausländerpolitik in Deutschland : Saisonarbeiter, Zwangsarbeiter, Gastarbeiter, Flüchtlinge, München, Beck, 2001 ; Bruno A.S., Rygiel P., Spire A., Zalc C., «Jugés sur pièce. Le traitement des dossiers de séjour et de travail des étrangers en France (1917-1984) ", Population, vol.61, n5-6, 2006, pp. 737-762.

36. Akoka K., L'évolution de la fabrique du réfugié à OFPRA de 1952 aux années 2000, thèse de sociologie en cours, Université de Poitiers, UMR 6588. 
heurte à un doute radical et une procédure de vérification rigoureuse. Les exigences et le contrôle institutionnel stimulent la créativité des demandeurs dans l'invention de moyens pour, tour à tour, y répondre et s'y soustraire. Dans l'instruction des demandes, la question de la crédibilité du récit et de l'établissement des faits occupe aujourd'hui une place tellement centrale que celle de la qualification juridique des faits s'en trouve parfois reléguée au second plan: "J'essaye plutôt de voir les faits. Et j'essaye de voir si ce qu'il raconte est vrai ou pas. Pendant l'entretien ce n'est jamais le côté juridique qui prime. C'est vraiment le côté récit. Le juridique, je le vois après. Surtout qu'en général c'est assez clair », m'explique cette instructrice de l'OFPRA alors qu'un instructeur du BAMF affirme même que " la question de la crédibilité commence déjà avant la présentation orale du récit. Ça commence déjà avec les papiers d'identité ». Les acteurs du dispositif d'asile affirment quasi unanimement que le droit d'asile est un droit très subjectif où le décideur ne peut, en l'absence de preuves matérielles et de témoins, que s'appuyer sur les déclarations de l'intéressé. Bien évidemment ceci met en cause la capacité décisionnelle des agents et, par voie de conséquence, la légitimité de décisions administratives et juridiques qui se fondent très largement sur l'intime conviction et le simple fait de croire ou ne pas croire ${ }^{37}$.

\section{Offre d'asile 38}

La procédure d'asile se présente désormais comme un processus lors duquel les uns exercent un pouvoir sur les autres qui, quant à eux, essayent en même temps de répondre aux exigences imposées et de se soustraire au contrôle.

Si les dispositifs d'asile nationaux et européen ont évolué dans un sens toujours plus restrictif, dissuasif et répressif, et que la prestigieuse mission d'accueil des réfugiés semble s'être mue en une obligation dérangeante, il faut tout de même prendre acte du fait qu'ils existent et fonctionnent encore. Bien qu'ils constituent avant tout des dispositifs de rejet plus que d'accueil, on persiste à acclamer l'idée initiale de protection. La « fiction juridique de l'asile ${ }^{39}$ » est donc maintenue en dépit de la faiblesse des taux d'accord. Au lieu de « laisser mourir le patient malade ", l'UE réalise depuis la fin des années quatrevingt-dix des efforts pour lui insuffler une nouvelle vie - et un nouveau sens. L'harmonisation des politiques d'asile au niveau européen constitue ici une préoccupation centrale : il s'agit de créer une situation où la demande d'asile

37. Voir par exemple : Greslier F., « La Commission des recours des réfugiés ou "l'intime conviction" face au recul du droit d'asile en France ", Revue européenne des migrations internationales, vol.23, n², 2007, pp. 107-133; Valluy J., Rejet des exilés. Le grand retournement du droit d'asile, op. cit.

38. Terme emprunté à Akoka K., Du réfugié au demandeur d'asile : construction de problèmes et transformations des catégories d'intervention à l'OFPRA, intervention au séminaire du GSPE à Strasbourg, 3 mai 2010.

39. Valluy J., "La fiction juridique de l'asile », Plein Droit, n63, décembre 2004, pp. 17-22. 
rencontre, au sein de l'UE, une offre d'asile qui serait la même dans chaque État membre. La possibilité de demander un statut de protection internationale peut en effet être conçue comme une opportunité juridique offerte par les pays d'accueil - la demande d'asile constituant un saisissement de cette opportunité.

La métaphore du «marché de l'asile » permet de s’interroger sur la manière dont l'offre et la demande d'asile se conditionnent mutuellement. Ainsi, les États européens se sont rendu compte que l'offre d'asile détermine en partie la demande. Une pratique décisionnelle généreuse envers un groupe particulier de demandeurs peut aboutir à une amplification de ce flux. L'offre d'asile, s'exprimant à travers le taux d'accord, constitue donc potentiellement un instrument de régulation des flux que certains États utilisent de manière plus affirmée et décomplexée que d'autres. Le BAMF est placé sous la tutelle du ministère de l'Intérieur qui lui communique des consignes précises, y compris sur la manière de considérer tel ou tel groupe de demandeurs. À l'aide de l'organe exécutif (le BAMF), le ministère essaye donc d'aiguiller la demande à travers l'offre d'asile. En France, on regarde d'un air sceptique ces pratiques allemandes. Un chef de section de l'OFPRA raconte l'anecdote suivante : lors d'une réunion consultative entre des représentants institutionnels de différents pays européens, le représentant du BAMF expliquait que le ministère venait de suspendre la considération des Yézidis d'Irak comme un groupe dont les membres ont automatiquement droit à une protection. Interrogé sur le motif de cette décision, il répondait, en riant mais de manière malgré tout affirmative : «Ah, c'est juste parce qu'on en avait trop ». Alors que l'intervention régulatrice du ministère sur les pratiques décisionnelles du BAMF est un fait notoire en Allemagne, l'OFPRA réclame et affirme son intégrale indépendance vis-à-vis de son ministère de tutelle. Les agents de l'OFPRA défendent unanimement le point de vue que le nombre d'accords prononcés dépend exclusivement de la demande, et de sa qualité. Le ministère de tutelle n'a, selon eux, ni ordres ni avis à donner sur les questions de fond de l'instruction. Seule la juridiction d'appel est compétente pour contrôler et remettre en cause les décisions administratives.

\section{Le contrôle décisionnel}

L'hypothèse prétendant que le taux d'accord serait utilisé, nonobstant les affirmations contraires de certains pays, comme moyen de dissuasion a notamment été défendue par Holzer et Schneider. « Puisque le nombre relatif d'accords a reculé drastiquement durant les années quatre-vingt [période de forte augmentation de la demande en Europe], ces affirmations ne peuvent être prises au sérieux. Cela supposerait que la qualité des requêtes aurait varié de façon proportionnelle 40 ». La question sous-jacente est bien entendu celle 
de l'existence ou non de quotas d'admission. Le soupçon selon lequel ces pratiques décisionnelles de l'OFPRA seraient soumises à des quotas émanant du ministère est très répandu dans les milieux associatifs et militants. Les agents de l'OFPRA, y compris ceux ayant antérieurement travaillé en association, rejettent vigoureusement cette "rumeur des quotas ». Les entretiens font cependant apparaittre l'existence d'une limite bien plus subtile et moins saisissable : « la barre ». Dans l'instruction des dossiers tangents, cette barre indique le niveau d'exigence, de tolérance, de sévérité ou de générosité. La large place occupée par la question de la crédibilité dans l'instruction des demandes d'asile rend les décisions malléables et la proportion relative de chaque type de décision ajustable. Le fait que la perception de la crédibilité soit intrinsèquement subjective anéantit la relative objectivité de la loi. «On sait ce qui relève du champ d'application de la Convention de Genève et après c'est surtout une question de faits établis ou pas. Le problème est de savoir où on met la barre ", me dit une instructrice française. Selon les propos des instructeurs, la hauteur à laquelle est placée cette barre dépend principalement d'une appréciation individuelle. Il existe cependant un contrôle par le supérieur hiérarchique institutionnel, qui révise les propositions de décision de ses agents, ainsi que par la CNDA, susceptible d'annuler la décision de l'agent administratif. Alors que l'Allemagne ne dispose pas non plus de quotas officiels, le contrôle décisionnel interinstitutionnel s'exerce sur un mode différent. L'ajustement du taux d'accord est avant tout assuré par une doctrine institutionnelle très formalisée et précise. En fonction de consignes ministérielles, cette doctrine est rédigée et actualisée par la centrale de pilotage du BAMF 41 et transmise aux instructeurs sous forme de « lignes directrices sur les pays d'origine » (Herkunftsländer-Leitsätze). Ceux-ci sont tenus de rendre leurs décisions conformément à ces principes doctrinaux et d'utiliser dans la rédaction de leurs décisions des paragraphes préformulés mis à disposition par la centrale. La doctrine constitue le principal instrument de contrôle des décisions, dans la mesure où elle indique la limite qui sépare les dossiers acceptés des dossiers rejetés.

Nous avons ainsi affaire à deux modes de contrôle décisionnel intra-institutionnel différents, l'un personnel (contrôle par le chef de section) et l'autre impersonnel (contrôle par la doctrine écrite). Il apparaît dès lors intéressant de constater que chaque institution essaye, par son moyen de contrôle privilégié, d'orienter les décisions des instructeurs dans un sens ou dans l'autre. Les instructeurs français expriment une frustration liée au fait de ne pas pouvoir reconnaître plus de personnes, ce qu'ils expliquent par la mauvaise qualité de

allen Ländern drastisch zurückgegangen ist, sind diese Beteuerungen kaum ernst zu nehmen. Sie würden voraussetzen, dass sich die Qualität der Gesuche in gleichem Ausmaß verändert hätte. ». Holzer T., Schneider G., Asylpolitik auf Abwegen, Opladen, Leske + Budrich, 2002, p. 40 (traduction par l'auteure).

41. Le BAMF est une organisation décentralisée et se compose donc d'une centrale située à Nürnberg et de 22 bureaux excentrés situés dans les différents Länder. 
la demande d'un côté, et la réticence des chefs de section à approuver des propositions d'accord de l'autre. Selon les instructeurs, il est généralement plutôt difficile de "faire passer un accord » auprès du chef tandis que les propositions de rejet sont très rarement retoquées. De manière contraire, les instructeurs allemands regrettent que les lignes directrices les obligent parfois à prendre des décisions positives qu'ils trouvent injustifiées. Il apparaît dès lors que les dirigeants du BAMF incitent les instructeurs à une pratique décisionnelle plus généreuse tandis que ceux de l'OFPRA cherchent à juguler les velléités d'accord des instructeurs.

\section{Face à la mission de l'instruction}

La différence sensible qui se constate entre l'attitude que les instructeurs allemands et français déploient face aux demandeurs d'asile et face à l'injonction hiérarchique doit être interprétée à la lumière de leur profil socioprofessionnel exposé au début de l'article. En effet, les instructeurs de l'OFPRA font preuve d'une plus grande «indulgence » et compréhension à l'égard des demandeurs et sont parfois très affectés par les histoires qu'ils racontent. Ils rappellent souvent que leur véritable mission professionnelle réside dans la protection des réfugiés et non en leur rejet, ce qui explique leur propension à proposer des accords. Les instructeurs du BAMF cependant témoignent souvent d'un certain blasement professionnel, d'une lassitude à entendre des histoires incroyables, voire même d'une vexation face aux mensonges des demandeurs. Partant du principe que la plupart des histoires sont fictives, leurs contenus violents et dramatiques les affectent relativement peu. Bien que les instructeurs allemands se réjouissent quand ils peuvent reconnaitre un demandeur qui leur a paru authentique, le rejet massif des «fraudeurs » est accepté comme faisant partie intégrante de leur tâche professionnelle. Ainsi, certains parmi eux disent «souffrir de l'injustice de devoir [en raison de la consigne institutionnelle] fournir une protection à des gens qui ne la méritent pas ».

Le critère de l'ancienneté dans le métier - qui est évidemment lié à celui de l'âge - apparaît comme le plus explicatif des différences esquissées ci-dessus. $\mathrm{Au}$ fil d'une expérience professionnelle prolongée, les instructeurs gagnent une distance émotionnelle par rapport à la matière humaine qu'ils traitent, distance qui peut également prendre la forme d'une amertume et d'un désappointement. Soulignons cependant qu'il ne s'agit en aucun cas d'un automatisme et que le matériau empirique contient des contre-exemples. En second lieu, le critère de la formation et la sensibilité initiale à la problématique de l'asile permettent également d'expliquer l'écart entre les deux attitudes décrites ci-dessus. Et finalement, le critère du genre n'est probablement pas insignifiant. Selon la rumeur, les chefs de section à l'OFPRA sont encore plus regardants sur les accords proposés par des instructrices que l'on soupçonne plus facilement de céder à un sentiment de compassion. Au BAMF cependant, certains 
instructeurs ayant un penchant pour les décisions d'accord se voient stigmatisés comme «femmelettes » ou « couilles-molles».

Au-delà des différences mises en évidence ci-dessus, les instructeurs du BAMF et de l'OFPRA se ressemblent surtout dans leur manière d'instruire la demande d'asile. Tous connaissent les difficultés de la mission de tri qui leur incombe. Tous connaissent le tiraillement entre le rôle de juge, d'administrateur, de contrôleur, de confident et de protecteur. Le vécu des instructeurs, leur manière de faire et leur ethos professionnel ne peuvent être saisis qu'à travers une étude du dispositif d'asile «par le bas ». La procédure de sélection et les résultats qu'elle produit sont déterminés par une multitude de facteurs les instructeurs en constituent un. Leur rôle est d'autant plus déterminant que la question de la crédibilité prend souvent le pas sur celle de la qualification juridique des faits. Ce constat remet par ailleurs en cause l'efficacité de tentatives européennes d'harmoniser les pratiques décisionnelles des États membres par la création d'une loi commune. Bien que les instructeurs occupent donc un poste clé dans le processus décisionnel, leur marge de manœuvre et leur pouvoir discrétionnaire sont limités par le contrôle intra-institutionnel et juridictionnel. Finalement, il ne faut pas sous-estimer l'impact que les demandeurs exercent eux-mêmes sur le déroulement de leur procédure. En dépit de leur position de dominés, ils mobilisent des ressources propres et bénéficient souvent de l'aide d'un réseau d'acteurs bien développé. Beaucoup de choses se jouent donc en amont de l'étape institutionnelle. Il devient finalement apparent que les chercheurs n'en ont pas fini d'étudier le dispositif d'asile national et européen qui recouvre encore beaucoup de zones d'ombre demandant à être éclairées. 